\title{
Asymmetric exclusion process with next-nearest-neighbor interaction: some comments on traffic flow and a nonequilibrium reentrance transition
}

\author{
T. Antal ${ }^{1,2}$ and G. M. Schütz ${ }^{2}$ \\ 1 Département de Physique Théorique, Université de Genève, 1211 Genève 4, 24 quai Ernest Ansermet, Switzerland \\ ${ }^{2}$ Institut für Festkörperforschung, Forschungszentrum Jülich, 52425 Jülich, Germany
}

(November 8, 2018)

\begin{abstract}
We study the steady-state behavior of a driven non-equilibrium lattice gas of hard-core particles with next-nearest-neighbor interaction. We calculate the exact stationary distribution of the periodic system and for a particular line in the phase diagram of the system with open boundaries where particles can enter and leave the system. For repulsive interactions the dynamics can be interpreted as a two-speed model for traffic flow. The exact stationary distribution of the periodic continuous-time system turns out to coincide with that of the asymmetric exclusion process (ASEP) with discrete-time parallel update. However, unlike in the (single-speed) ASEP, the exact flow diagram for the two-speed model resembles in some important features the flow diagram of real traffic. The stationary phase diagram of the open system obtained from Monte Carlo simulations can be understood in terms of a shock moving through the system and an overfeeding effect at the boundaries, thus confirming theoretical predictions of a recently developed general theory of boundary-induced phase transitions. In the case of attractive interaction we observe an unexpected reentrance transition due to boundary effects.
\end{abstract}

PACS: 05.70.Ln, 64.60.Cn, 02.50.Ga

\section{INTRODUCTION}

One of the basic properties of many driven, interacting many-body systems is the occurrence of shocks. A shock in a system of classical flowing particles marks the sudden transition from a region of low density to a region of high density. A well-known example for a shock is the beginning of a traffic jam on a motorway where incoming cars (almost freely flowing particles in the low density regime) have to slow down very quickly over a short distance and then form part of the (high-density) congested region. A remarkable feature of such shocks is their stability over long periods of time, i.e., they remain localized over distances comparable to the size of particles. In some sense one may regard shocks as soliton-like collective excitations of the particle system.

Lattice gas models have proven to be excellent systems for the theoretical investigation of shocks and of the consequences of shocks for the collective behavior of the particle system. An interesting situation is the coupling of such a driven lattice gas system to external particle reservoirs 13 . It is intuitively clear that unlike in equilibrium systems, here the boundaries will play a decisive part in determining the bulk behavior of the system: Since the system is open at the boundaries, particles will flow in and out and the current will carry boundary effects into the bulk. Among the fundamental questions to ask in such a set-up is the stationary (i.e. long-time) behavior of the system as a function of the boundary densities. Numerical observations and mean-field based arguments show that varying boundary densities leads to boundary-induced phase transitions [3]. To understand why this happens it is clearly necessary to get insight into the collective behavior of the lattice gas and to investigate the role of the shocks.
The best-studied example is the one-dimensional asymmetric simple exclusion process (ASEP) [ [ 6], a hard-core lattice gas where each lattice site can be occupied by at most one particle and particles hop stochastically with constant bias to vacant nearest-neighbor sites. For this model not only the structure and motion of shocks is largely understood, but also the stationary phase diagram of the open system [2] and the exact particle-density profiles [7,8] are explicitly known as function of the external reservoir densities. Based on the exact solution of the ASEP and of a related lattice gas model [9] the role of shocks for the stationary phase of more generic open driven diffusive systems was elucidated [10,11]. This has led to a theory of boundary-induced phase transitions for one-component driven particle systems in one dimension [5], reviewed below. Of particular interest are systems where the stationary current $j(\rho)$ as a function of particle density $\rho$ has a single maximum, an example being traffic flow on single-lane or multi-lane highways 12,13]. The theory predicts a phase diagram with a first-order (non-equilibrium) phase transition between a low-density phase (LD) and a high density phase (HD) and a continuous (second-order) order transition from both phases to a maximal-current phase (MC), see Fig. 2 below. In the context of traffic flow the properties of the second-order transition suggest more efficient control mechanisms for avoiding jams [14].

The strength of the theory lies in the small number and generality of its assumptions. Hence it is tempting to test its validity in real traffic flow. The openness of the system models in- and outflow of cars on a road between two junctions. Indeed, the main features of the predicted first-order transition have been observed using data collected on a German motorway [14,15. The second-order transition has been confirmed by Monte-Carlo simula- 
tions of a suitably modified Nagel-Schreckenberg model for traffic flow, originally introduced only for periodic boundary conditions 16 18]. Since to our knowledge the data necessary to test the existence of the second-order transition in real traffic are not available at present, independent investigations of other traffic flow models are required to establish the presence of such a transition. We consider here a continuous-time exclusion model with additional short-range interaction on top of the pure hardcore repulsion of the usual ASEP. The model is designed to be exactly solvable like the ASEP (to some degree) on the one hand and to be somewhat more realistic in describing real traffic than the ASEP on the other hand. However, given the wide range of experimental applications of hard-core lattice gases which comprise phenomena as diverse as the kinetics of protein synthesis [1], diffusion in thin channels [19], or polymer reptation [20], broader relevance of our model is to be expected. Indeed, in order to obtain a more complete picture we also analyse a similar model with attractive next-nearestneighbour interaction. This model is not related to traffic flow, but describes driven hard-core particles with attractive short-range interaction. To our knowledge, this is the first investigation of the steady-state selection of a driven diffusive system with this type of interaction.

The paper is organized as follows. In Sec. II we comment on some of the requirements of traffic flow modeling and we describe our model. This model is a special case of the driven diffusive systems studied some while ago by Katz, Lebowitz and Spohn 21. We explain in which respect this model is more realistic for traffic flow than the standard ASEP which is a limiting case of our lattice gas. This phenomenological explanation is then confirmed by the calculation of the exact flow diagram, i.e. the stationary bulk current as a function of the density. In this section the emphasis is on bulk properties and hence we investigate the system with periodic boundary conditions in the thermodynamic limit. In Sec. III we review some details of the theory of boundary-induced phase transitions of Ref. [10] and we introduce the boundary dynamics for modeling the coupling of a finite system to boundary reservoirs of constant density. We give an exact solution for the stationary distribution along the line in the phase diagram corresponding to equal boundary densities (with proof postponed to the appendices) and discuss a mean-field analysis of the full phase diagram. In Sec. IV] we present a mean-field analysis of the full phase diagram and discuss Monte Carlo data for the phase transition lines while in Sec. V we perform a similar analysis for the model with attractive interaction. Finally we summarize our findings and present some concluding remarks (Sec. VI). Technical details of the derivation of exact results are presented in the appendices.

\section{ASEP WITH NEXT-NEAREST-NEIGHBOR INTERACTION - SOME COMMENTS ON MODELLING TRAFFIC FLOW}

The ASEP is a stochastic lattice gas of hard-core particles with biased particle hopping. Particles hop with exponential waiting-time distribution with parameter 1 to their nearest-neighbor site to the right, provided this site is empty. If the site is occupied, the hopping attempt is rejected. Symbolically one may represent these stochastic dynamics as follows

$$
A \emptyset \rightarrow \emptyset A \text { with rate } 1 .
$$

Here $A$ represent a particle and $\emptyset$ represents the vacant neighboring site. Even though this stochastic process is too simple to be a realistic model for traffic flow, some qualitative features of real traffic [22,23] can already be seen: Shocks exist and the stationary current $j(\rho)=\rho(1-\rho)$ as a function of particle density $\rho$ has a single maximum. An apparently unrealistic feature of the particle distribution is the absence of correlations in the steady state which are seen in more sophisticated traffic flow models [17,18]. An unrealistic feature of the currentdensity relation (known in traffic engineering as flow diagram) is the reflection symmetry w.r.t. the maximalcurrent density $\rho^{*}=1 / 2$ and its rounded shape close to the maximum.

The basic mechanisms which determine traffic flow appear to be firstly the competition between the desire to reach an optimal speed while keeping a (velocitydependent) safety distance. When the traffic density becomes sufficiently large this distance cannot be kept anymore and the speed has to be reduced. As a consequence the current drops at some density $\rho^{*}$. Secondly, there is a certain amount of randomness due to variations in individual drivers behavior. This "noise" necessitates a statistical description of traffic flow phenomena.

Various one-dimensional lattice gas models which incorporate these mechanisms in different manners have been proposed [12]. The following is part of the picture that emerges:

(i) The existence of a shock is generic and appears to be the consequence of the non-linear current-density relation [5].

(ii) The symmetric shape of the current-density relation results from particle-hole symmetry and is a feature of models in which cars move with constant probability or rate, independently of the environment beyond the nearest neighbor site to which they move. This is an unrealistic assumption since clearly car drivers slow down when they see a slowly moving car already some distance ahead. They do not just perform an emergency stop when the car is immediately in front of them. Numerical and analytical results for models 16, 18,24 which allow for a reduction of speed that depends on the occupation of sites further ahead show an asymmetric currentdensity relation resembling the shape of the currentdensity relation of real traffic. In these models speed 
is implemented by jumps over a variable number of lattice sites.

(iii) The unrealistically round shape of the currentdensity relation at $\rho^{*}$ is specific for the ASEP. Deterministic exclusion processes with parallel update 25 30 also show a symmetric current-density relation with one maximum, but the derivative of the current is discontinuous at the maximal-current density $\rho^{*}$, in this respect resembling the shape of the current in real traffic 22] and of more realistic traffic flow models [16]. Increasing the hopping probability in a discrete-time process towards deterministic hopping, leads to an increasingly sharp jump in the current derivative at $\rho^{*}$ 17. Hence the rather broad exponential waiting-time distribution of the standard ASEP seems to be responsible for the round shape of the current-density relation at $\rho^{*}$. In terms of the motion of a single particle these dynamics correspond to an overestimated single-particle diffusion coefficient.

(iv) For parallel update, but not for sublattice parallel update, increasing the hopping probability strengthens antiferromagnetic particle correlations 18,28, i.e., cars are less likely to be found close to each other than some distance apart.

In order to further investigate this picture and to disentangle the various effects associated with a small diffusion coefficient and with speed-reduction respectively it would be interesting to study both ingredients separately for models in which the stationary distribution can be calculated exactly. A small single-particle diffusion coefficient can be implemented in discrete-time models choosing the hopping probability close to one (low noise). In the single-speed ASEP this has been shown to lead to the (almost) discontinuous behavior of the current derivative discussed above. The numerical results on the effect of a small diffusion coefficient on correlations are inconclusive. In our toy model we keep the exponential waiting-time distribution (large diffusion coefficient), but introduce a next-nearest-neighbor interaction which in the repulsive case models slowing down of a car if the next-nearest-neighbor site is occupied as well. A particle hops to the right with rate $r$ if the next-nearest-neighbor site is empty and with rate $q$ if it is occupied:

$$
\begin{array}{ll}
A \emptyset \emptyset \rightarrow \emptyset A \emptyset & \text { with rate } r \\
A \emptyset A \rightarrow \emptyset A A & \text { with rate } q .
\end{array}
$$

The condition $q<r$ models slowing-down, in the limiting case $r=q$ one recovers the usual ASEP. For $q>r$ this model has not an interpretation as traffic model, but may be regarded as describing hard-core particles with attractive short-range interaction which are driven by an external field.

This model is a special case in the class of driven diffusive systems investigated in Ref. 21]. On a ring with $N$ sites with periodic boundary conditions the stationary distribution turns out to be given by the equilibrium distribution of the one-dimensional Ising model. Each state of the system is defined by the set of occupation num- bers $\underline{n}=\left\{n_{1}, \ldots, n_{N}\right\}$ with $n_{i}=0,1$. The stationary probability of finding a state $\underline{n}$ is given by

$$
P^{*}(\underline{n})=\frac{1}{Z_{N}}\left(\frac{q}{r}\right)^{\sum_{i=1}^{N}\left(n_{i} n_{i+1}+h n_{i}\right)} .
$$

Here $Z_{N}$ is the partition function and the "chemical potential" $h$ parametrizes the conserved bulk density $\rho$. This grand-canonical distribution is a non-equilibrium stationary state, i.e., it is invariant under the stochastic time evolution, but it does not satisfy detailed balance with respect to the dynamics. It is interesting to notice that correlations are non-vanishing and, in the repulsive case $q<r$ which corresponds to speed reduction, become antiferromagnetic. In fact, the stationary state is identical to that of the discrete-time ASEP with parallel update [18,31] for hopping probability $p=1-q / r$.

According to the dynamics described above the local density satisfies the continuity equation

$$
\frac{d}{d t}\left\langle n_{i}\right\rangle=\left\langle j_{i-1}\right\rangle-\left\langle j_{i}\right\rangle
$$

with the current

$$
\left\langle j_{i}\right\rangle=\left\langle n_{i}\left(1-n_{i+1}\right)\left[q n_{i+2}+r\left(1-n_{i+2}\right)\right]\right\rangle
$$

between sites $(i, i+1)$. The stationary particle current $j=\left\langle j_{i}\right\rangle$ is constant and is readily calculated using standard transfer matrix techniques for the one-dimensional Ising model 32] (see Appendix A). In the thermodynamic limit $N \rightarrow \infty$ one finds the exact current density relation

$$
j=r \rho\left[1+\frac{\sqrt{1-4 \rho(1-\rho)(1-q / r)}-1}{2(1-\rho)(1-q / r)}\right]
$$

shown in (Fig. 1) in the repulsive case.

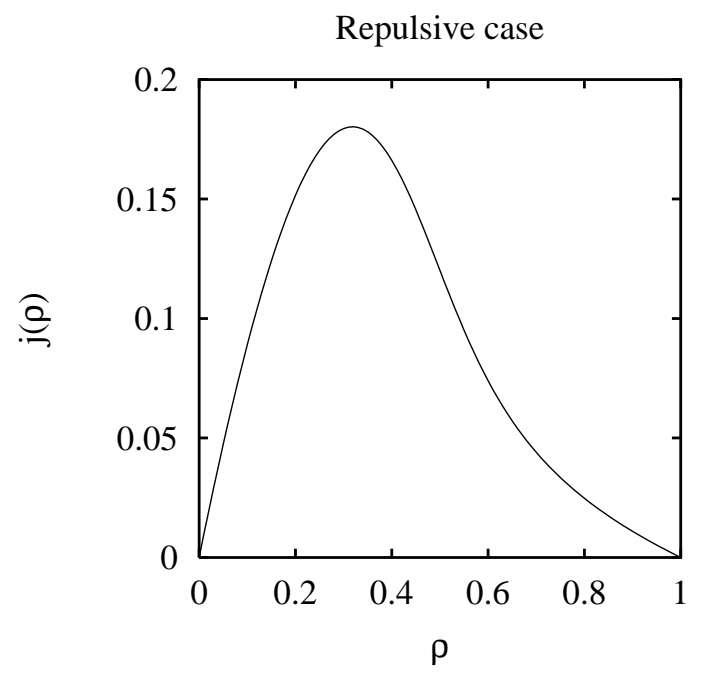

FIG. 1. Stationary current $j$ as a function of the density $\rho$ for $r=1, q=0.1$ (repulsive interaction). 


\section{OPEN BOUNDARIES}

The analysis of the previous section yields the bulk properties of the lattice gas at a given density. Now we address the question of the steady-state selection of the system with open boundaries, i.e., we investigate the bulk density of an open system coupled at both ends to reservoirs of different fixed boundary densities. Translated into traffic language, open boundaries correspond to traffic junctions at the ends of a road where particles (cars) enter or leave the road respectively with certain fixed attempt rates, a situation envisaged with different aims already in 33] for the ASEP with parallel update. As a result of the coupling to reservoirs a non-trivial stationary density profile in the vicinity of the boundaries will emerge and the (spatially constant) bulk-density will be a function of the two reservoir densities.

There are two distinct mechanisms at work. Firstly, because of the particle interaction, coupling of a semiinfinite system to a reservoir will generically lead to some discontinuous behavior of the stationary distribution close to the boundary. The boundary represents an inhomogeneity of the system since the interaction of the particles with the fixed boundary leads to different dynamics than that which results from the interaction of particles among themselves. This is a non-universal phenomenon which depends on the precise nature of the coupling mechanism and on the nature of the particle interaction. For short-range hopping and systems with shortrange stationary bulk correlations one expects the following picture: Coupling of a semi-infinite system at site 1 to a reservoir of constant density $\rho_{L}$ will give rise to a non-universal boundary density profile starting at $\rho_{L}$ and approaching (on the scale of lattice units) some bulk density $\rho_{-}$which is a non-universal function of $\rho_{L}$ (Fig. 2). A similar picture holds for coupling of a semi-infinite system at the right boundary where particles flow out of the system into the reservoir. Here the bulk density $\rho_{+}$may change close to the boundary to the reservoir density $\rho_{R}$. Superimposed on this non-universal boundary structure is a universal behavior which depends only the effective boundary densities $\rho_{-}, \rho_{+}$close to, but not at the boundaries. The theory of boundary induced phase transition [10] describes the stationary phase diagram in terms of these effective boundary densities.

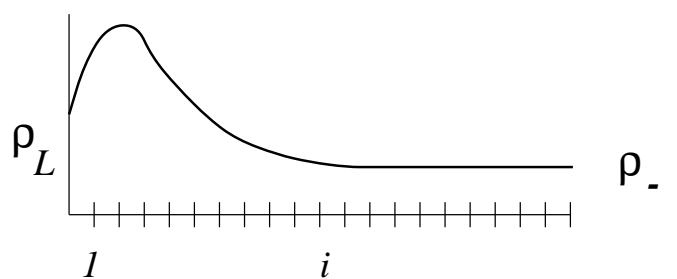

FIG. 2. Coupling of a semi-infinite driven particle system to a reservoir of constant particle density $\rho_{L}$. The (interpolated) density profile $\rho_{i}(i=1,2, \ldots)$ approaches some constant value $\rho_{-}$after some finite distance from the boundary.

\section{A. Theory of boundary-induced phase transitions}

We review only the principal ideas of this theory [5] which is based on an interplay of the collective velocity

$$
v_{c}=\frac{\partial j}{\partial \rho}
$$

of the lattice gas and the shock velocity

$$
v_{s}=\frac{j_{+}-j_{-}}{\rho_{+}-\rho_{-}}
$$

of a shock with limiting densities $\rho_{+}$and $\rho_{-}$and with limiting currents $j_{+}$and $j_{-}$to the right and to the left respectively.

The collective velocity is the velocity of the center of mass of a local perturbation in a homogeneous stationary background (Fig. 3a). It is positive for background density $\rho<\rho^{*}$, but becomes negative for $\rho>\rho^{*}$. In this case the perturbation creates a back-moving traffic jam, which leads to a negative center-of-mass velocity, even though all individual particles move with positive velocity. In terms of traffic flow one might think of such a perturbation as being a car which has just entered a major throughway from some side road.

The shock velocity describes the motion of the shock which performs, due to fluctuations, a biased random walk with velocity $v_{s}$ (Fig. $3 \mathrm{~b}$ ). If the incoming current $j_{-}$exceeds the outgoing current $j_{+}$, the shock velocity is negative. This is analogous to the back-moving shock of a traffic jam for sufficiently high incoming traffic flow.

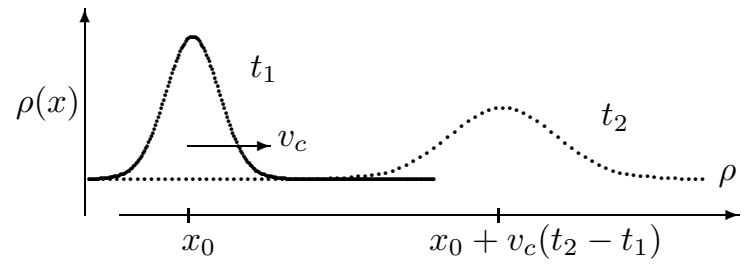

(a)

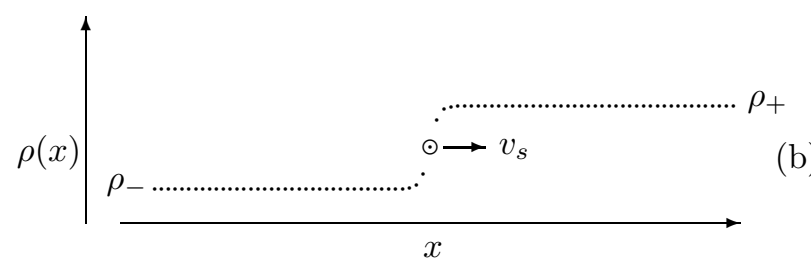

FIG. 3. (a) Diffusive spreading of a density perturbation in the stationary state at two times $t_{2}>t_{1}$. The collective velocity describes the motion of the center of mass of the perturbation. (b) Motion of a shock. To the left of the domain wall particles are distributed homogeneously with an average density $\rho_{-}$. To the right of the domain wall the background density is $\rho_{+}>\rho_{-}$

To get an intuitive understanding how these velocities determine the stationary phase diagram of a driven system coupled to boundary reservoirs let us assume $\rho_{ \pm}=\rho_{R, L}$. We consider first $\rho_{+}>\rho^{*}$ where $\rho^{*}$ is the density where the current takes its maximal value $j^{*}$. 
To make the argument more transparent we also assume that particles hop only to the right and that initially the lattice is empty. Because of ergodicity the stationary distribution is independent of the initial state and hence this assumption involves no loss of generality. Consider now the time evolution of the averaged density profile in a large system, starting from the empty lattice. We start the discussion by assuming the left boundary density to be very low.

(i) As time proceeds, particles from the left reservoir will enter the system and (possibly after some distance describing the non-universal boundary layer) create a region of constant density $\rho_{-}$. This region decays to the right to zero, because after a finite time the rightmost particles will have traveled only a finite distance. Eventually however, after a time which is of the order of system size, the rightmost particles will hit the right boundary with the reservoir of density $\rho_{+}$. This reservoirs makes it more difficult for particles to travel further and hence creates a little traffic jam. The result is a shock profile, with shock densities $\rho_{-}$on the left and $\rho_{+}$to the right resp. like in Fig. $3 \mathrm{~b}$. [For the sake of the argument one could have chosen such an initial state. The reason for choosing an empty initial state becomes clear below.] The decisive question is now how this shock profile evolves in time. According to (9) the shock velocity under the circumstances described here is positive, simply because the incoming current of particles $j_{-}$is less than the outgoing current $j_{+}$for sufficiently small left boundary density. Hence, even though a shock forms by fluctuations, it has an average drift towards the right boundary. Hence the system remains in the low density (LD) regime with bulk density $\rho=\rho_{-}<\rho^{*}$.

(ii) The situation changes when the left boundary density takes a value such that the incoming current $j_{-}$ equals the outgoing current $j_{+}$. In this case the shock velocity vanishes and the shock performs an unbiased random walk over the lattice. Hence the density profile may be regarded as being composed of two stationary domains with densities $\rho_{-}$and $\rho_{+}$, separated by a "domain wall" which is the sharp transitional region of the shock. Since the shock motion is unbiased, the stationary probability of finding the shock is constant in space. This leads to an equal superposition of shock profiles and hence to a linearly increasing stationary density profile. In analogy to first order equilibrium phase transitions where a domain wall separates regions of coexisting equilibrium regimes, we call the line defined by $j_{+}=j_{-}$and $\rho_{-}<\rho^{*}$, $\rho_{+}>\rho^{*}$, a first order phase transition line.

(iii) This line marks the transition to a high-density phase (HD) with bulk density $\rho=\rho_{+}>\rho^{*}$ since for even higher left boundary density the incoming current into the shock exceeds the outgoing current, and according to (9) the shock moves to the left. This leaves the bulk in the high-density regime determined by the coupling to the right boundary reservoir. At the first-order transition line the stationary bulk density is discontinuous, it jumps from $\rho_{-}$to $\rho_{+}$.
Next we consider the case of low right boundary density $\rho_{+}<\rho^{*}$. For definiteness we choose $\rho_{+}=0$. The essential part of the following discussion, the explanation of the occurrence of a continuous phase transition, is unaffected by this choice. Again we start the discussion with the empty lattice.

(i) As argued above, after some initial time the system will have filled up to a bulk density $\rho=\rho_{-}$. Since $\rho_{+}=0$, particles hitting the right boundary can leave the system without creating a shock. As a result, the system is in the low-density phase. [For $\rho_{+}>0$ a shock could form, but would have positive velocity under the circumstances considered here. Hence also in this case the system is in the low-density phase.] Now we examine the seemingly trivial reason why an increase in the left boundary density leads to an increase in the bulk density. Above we simply claimed this to be true on the basis of plausibility. Here we support this claim with an argument that becomes important below. Suppose we create a little perturbation at the left boundary by injecting an extra particle (on top of those particles that are injected anyway from the reservoir). This creates a perturbation which, by definition of the collective velocity, travels with $v_{c}>0$ into the bulk and leads to a local increase of the density, moving away from the boundary and spreading out as time goes on. The collective velocity is positive, since by assumption we have a background density $\rho_{-}<\rho^{*}$ and hence the current as a function of the density has positive slope. The point is that maintaining such a perturbation (which corresponds to increasing the left reservoir density permanently) leads to a permanent additional flow of particles into the bulk and hence to the anticipated increase of the stationary bulk density.

(ii) It is now clear that this argument holds only as long as $\rho_{-}<\rho^{*}$. Assume now $\rho_{-}=\rho^{*}$. Following the reasoning above this results in a maximal-current bulk density $\rho^{*}$. However, if the left reservoir density increases beyond the maximal current density, the collective velocity becomes negative. No extra particles flow into the system, which therefore remains in its bulk at the maximal current density $\rho^{*}$. The system is now in the maximal current phase for all $\rho_{-}>\rho^{*}$ and $\rho_{+}<\rho^{*}$. This transition is continuous, the bulk density approaches $\rho^{*}$ smoothly from below. Intuitively this phenomenon may be understood as "overfeeding" [7]. The injected particles act as blockages for further incoming particles, leading to a back-moving traffic jam at the origin. This increased density at the origin blocks further injection attempts and prevents the actual increase of the current.

Finally, using similar arguments, one can show that the transition from the high-density phase to the maximal current phase is also continuous.

To summarize, the theory predicts a first-order transition along the line defined by $j_{+}=j_{-}$and $\rho_{-}<\rho^{*}$, $\rho_{+}>\rho^{*}$ where the stationary bulk density jumps from $\rho_{-}$ (low density phase LD) to $\rho_{+}$(high density phase HD). On the phase transition line the stationary density is linearly increasing from $\rho_{-}$to $\rho_{+}$. From both phases there 
is a continuous phase transition to the maximal current phase MC defined by $\rho_{-}>\rho^{*}$ and $\rho_{+}<\rho^{*}$. In this phase the bulk density takes the maximal current value $\rho=\rho^{*}$. These rules are encoded in an extremal principle for the current

$$
j= \begin{cases}\max _{\rho \in\left[\rho_{R}, \rho_{L}\right]} j(\rho) & \text { for } \rho_{L}>\rho_{R} \\ \min _{\rho \in\left[\rho_{L}, \rho_{R}\right]} j(\rho) & \text { for } \rho_{L}<\rho_{R}\end{cases}
$$

derived in 11. It is worthwhile pointing out that this dynamical theory explains in mesoscopic terms the predictions one would obtain by viewing the system from a coarse-grained hydrodynamic view-point [3]. At the same time, verification of this scenario suggests the validity of the hydrodynamic approach to the description of the large-scale dynamics of the particle system 34 by using the exact current-density relation.

\section{B. Coupling to boundary reservoirs}

To verify this scenario which correctly describes the exactly solvable ASEP with open boundaries one has to investigate our model in terms of the effective boundary densities $\rho_{-}, \rho_{+}$. Given two reservoir densities there is no general recipe how to eliminate the non-universal boundary effects that result in the effective boundary densities $\rho_{-}, \rho_{+}$. Hence these quantities are not easy to control. Ideally, for purposes of theoretical investigation, one would like to construct an injection and absorption mechanism which leads to a constant density profile for a semi-infinite system so that $\rho_{L}=\rho_{-}$and $\rho_{R}=\rho_{+}$, i.e. the effective boundary densities are identical to the actual control parameters of the model.

Here we choose an injection mechanism where the particles on the lattice interact with the reservoir particles in the same way as among each other. We define two injection rates from the reservoir at the left boundary:

- injection at site 1 if site 2 is occupied $|\emptyset A \rightarrow| A A$ with rate $\alpha_{1}$

- injection at site 1 if site 2 is empty $|\emptyset \emptyset \rightarrow| A \emptyset$ with rate $\alpha_{2}$

and two new hopping rates at the right boundary:

- hopping from site $N-1$ to site $N$ $A \emptyset|\rightarrow \emptyset A|$ with rate $\beta_{1}$

- hopping out from site $N$ (absorption) $A|\rightarrow \emptyset|$ with rate $\beta_{2}$.

These four hopping rates are those that would be affected by the interaction with particles in the reservoirs. The rates $\alpha_{i}$ ( $\beta_{i}$ resp.) have now to be determined as functions of the left (right) reservoir density. This can be illustrated e.g. for the injection process with rate $\alpha_{1}$. We imagine the reservoir to include a site 0 of the chain. The injection rate into the first site is defined by the (stationary) average occupation $\rho_{L}$ of the imaginary site 0 , but with the condition that the first site is empty and the second site is occupied. Considering the zeroth, the first and the second site as three neighboring sites of an infinite chain, this conditional probability can be expressed readily as correlations in the stationary state of an infinite chain. Thus we find $\alpha_{1}=q\langle 101\rangle /\langle 01\rangle$ where expectation values like $\langle 101\rangle=\left\langle n_{i}\left(1-n_{i+1}\right) n_{i+2}\right\rangle$ are calculated in the thermodynamic limit of the distribution (4) with density $\rho=\rho_{L}$. The case of $\alpha_{2}$ is entirely similar and one finds $\alpha_{2}=r\langle 100\rangle /\langle 00\rangle$.

For the calculation of the right boundary rates we note that the probability of jumping from the site $N-1$ to the site $N$ is affected by the average occupation of the imaginary reservoir site $N+1$. With the jump condition that site $N-1$ is occupied and site $N$ is empty one finds $\beta_{1}=(r\langle 100\rangle+q\langle 101\rangle) /\langle 10\rangle$. The case of $\beta_{2}$ is similar but one has to take into account the conditional probability of the occupation of two imaginary reservoir sites $N+1$ and $N+2$. This gives $\beta_{2}=(r\langle 100\rangle+q\langle 101\rangle) /\langle 1\rangle$. One has to determine these correlations in an infinite chain with density $\rho_{R}$. These rates can be expressed as a function of the density through the form of the current and we find:

$$
\begin{aligned}
& \alpha_{1}=q \frac{\langle 101\rangle}{\langle 01\rangle}=q \frac{\langle 10\rangle_{\rho_{L}}}{1-\rho_{L}} \\
& \alpha_{2}=r \frac{\langle 100\rangle}{\langle 00\rangle}=r \frac{\langle 10\rangle_{\rho_{L}}}{1-\rho_{L}} \\
& \beta_{1}=\frac{r\langle 100\rangle+q\langle 101\rangle}{\langle 10\rangle}=\frac{j\left(\rho_{R}\right)}{\langle 10\rangle_{\rho_{R}}} \\
& \beta_{2}=\frac{r\langle 100\rangle+q\langle 101\rangle}{\langle 1\rangle}=\frac{j\left(\rho_{R}\right)}{\rho_{R}}
\end{aligned}
$$

with

$$
\langle 10\rangle_{\rho}=\langle 01\rangle_{\rho}=(1-\rho)\left(1-\frac{j(\rho)}{r \rho}\right)
$$

For $\rho_{L}=1$ we use the limiting values $\alpha_{1}=q, \alpha_{2}=r$, and for $\rho_{R}=0$ we use $\beta_{1}=\beta_{2}=r$. Together with the bulk hopping rates $r, q$ the dynamics of the model is now completely defined.

Before discussing the full phase diagram we consider the case of equal boundary densities $\rho=\rho_{R}=\rho_{L}$. Somewhat surprisingly, it turns out that we can actually obtain the full stationary distribution of the process:

$$
P^{*}(\{n\})=\frac{1-\rho}{\lambda_{1}^{N-1}}\left(\frac{q}{r}\right)^{\sum_{i=1}^{N-1} n_{i} n_{i+1}} z^{\sum_{i=2}^{N-1} n_{i}}\left(\lambda_{1}-1\right)^{n_{1}+n_{N}}
$$

with the eigenvalue $\lambda_{1}$ of the transfer matrix of the onedimensional Ising model and the "fugacity" $z=e^{-\beta h}$ (Appendix A). Stationarity of this distribution can be proved by writing the time evolution operator of the 
process in a quantum Hamiltonian formalism (see appendix B). Like in the periodic case, the exact stationary non-equilibrium distribution of the open system with equal boundary densities is the equilibrium distribution of an Ising chain of length $N$, but with boundary fields $g\left(n_{1}+n_{N}\right)$ rather than with the Ising coupling $n_{N} n_{1}$.

Moreover, with the transfer matrix formulation of the Ising model it is straightforward to show that the density profile is constant. The theoretical scenario described above then suggests the identification $\rho_{-}=\rho_{L}$ for $\rho_{L}<\rho^{*}$ and $\rho_{+}=\rho_{R}$ for $\rho_{R}>\rho^{*}$. For $\rho_{L}>\rho^{*}$ or $\rho_{R}<\rho^{*}$ respectively constant profiles are not stable with respect to fluctuations and the relationship between the reservoir densities and the effective boundary densities may be more subtle (see below).

\section{PHASE DIAGRAM FOR REPULSIVE INTERACTION}

Having defined the model and given the theoretical background we are now set to investigate the phase diagram of the system. We consider first the traffic flow scenario (repulsive interaction).

\section{A. Mean field}

Unfortunately the exact stationary distribution of the open system for different boundary densities does not have a simple form. However, also the density in the open system satisfies a continuity equation of the form (5), with the boundary currents

$$
\begin{aligned}
& j_{0}=\alpha_{1}\left\langle\left(1-n_{1}\right) n_{2}\right\rangle+\alpha_{2}\left\langle\left(1-n_{1}\right)\left(1-n_{2}\right)\right\rangle \\
& j_{N-1}=\beta_{1}\left\langle n_{N-1}\left(1-n_{N}\right)\right\rangle \\
& j_{N}=\beta_{2}\left\langle n_{N}\right\rangle
\end{aligned}
$$

Using also the expression (6) for the bulk current one can obtain the stationary density profile in a mean field approximation by neglecting the correlations in the expectation values. Stationarity implies equal current everywhere in the lattice, $\left\langle j_{i}\right\rangle=j$. This gives rise to the bulk recursion relation

$$
\rho_{i}=\frac{j}{\left(1-\rho_{i+1}\right)\left(q \rho_{i+2}+r\left(1-\rho_{i+2}\right)\right)}
$$

for the density profile. The boundary values of the recursion are determined by the boundary currents (17) (19).

Because of the interaction with the boundary there are two possibilities to perform a mean-field analysis. The most straightforward choice would be to choose the boundaries rates (11) - (14) and analyze the mean field phase diagram in terms of the reservoir densities $\rho_{R, L}$. For a given choice of current one can draw a density profile and read off the various phases. However, it turns out that within such an approximation scheme a constant density profile cannot be achieved even if $\rho_{R}=\rho_{L}$ and that the boundary densities are not equal to the reservoir densities. As a result, the mean field phase diagram obtained in this way differs considerably from the theoretical expectation. Within this approximation the $\mathrm{MC}$ phase disappears for $r / q$ smaller than $\approx 0.6$.

From a theoretical point of view it is more natural to neglect the correlations even in the expressions of the boundary rates in terms of the Ising expectation values. This gives rise to simple expressions of the boundary rates in terms of the reservoir densities. Using the boundary conditions $\rho_{0}=\rho_{L}$ and $\rho_{N+1}=\rho_{N+2}=\rho_{R}$ extends the validity of the recursion (20) to the range $0 \leq i \leq N$. In this way $\rho_{N}$ can be expressed as a function of $\rho_{R}$ and $j$ and this was the reason of choosing the direction of the recursion from the right to the left. It is easy to see that for a semi-infinite system there are the constant solutions $\rho_{i}=\rho_{L}$ and $\rho_{i}=\rho_{R}$ resp. with the mean field current

$$
j^{M F}(\rho)=\rho(1-\rho)(q \rho+r(1-\rho))
$$

Like in the exact solution this is also a solution of the finite system with equal boundary densities. Therefore we shall discuss this mean-field approach in some more detail.

For general boundary densities we did not find a solution of the recursion (20) in closed form, but it is easily solved numerically on a computer. It is sufficient to choose a density $\rho_{R}$ and a current $j$ and apply the recursion relation for drawing the density profile. If any of $\rho_{i}$ 's is smaller than 0 or greater than 1 then there is no solution corresponding to these values of $\rho_{R}$ and $j$ at this system size since any physical solution has to satisfy $0 \leq \rho_{i} \leq 1$ for all $1 \leq i \leq N$. For fixed $\rho_{R}, \rho_{L}$ and length $N$ this requirement fixes the current $j$. In this way one can map out the whole phase diagram, finding the corresponding current for all values of $\rho_{L}$ and $\rho_{R}$. For sufficiently large $N$ the size dependence is negligibly small.

We have chosen $r=1.0$ and $q=0.1$. The low- (LD) and the high-density (HD) phases resp. are easy to distinguish as the bulk density is equal to one of the end densities. This allows us to identify $\rho_{-}=\rho_{L}$ and $\rho_{+}=\rho_{R}$. At the other end oscillatory behavior is observed. This does not happen in the usual ASEP with open boundaries [7, 8 , but has been observed in an ASEP with particles covering more than one lattice site [1]. The maximal mean field current accessible at given parameters $r$ and $q$ and the corresponding bulk density define the maximal current phase MC. The location of these phases in the parameter space differs from those obtained from theoretical scenario reviewed above (Fig. A) by using the exact value (7) of the current. Further analysis shows that the discrepancy increases with increasing repulsion. 


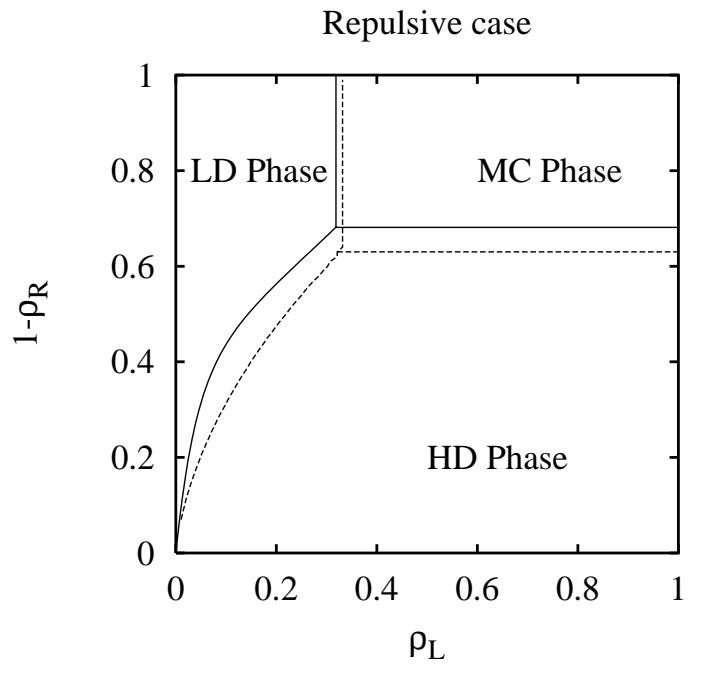

FIG. 4. Mean field phase diagram (broken curves) and theoretical phase transition lines (full curves) for repulsive interaction.

\section{B. Monte Carlo results}

In order to check the phase diagram based on the theory of boundary induced phase transition described above we have performed Monte Carlo simulations of the model.

The boundary rates are determined as explained above to simulate given left and right densities. During one MC step one of $N+1$ sites is chosen randomly ( $N$ is the size of the system and the +1 is for the jumping into the system) and if there is a particle then it can hop with a probability given by the hopping rates. The initial configuration was an empty lattice. The required time to reach the stationary state for given system size can be determined by investigating the time dependence of the current and the bulk density (the density in the middle of the system). The order parameter which is the stationary value of the bulk density obtained as an average over $10^{7}$ MC steps is the best indicator of the phase transition lines. At the first order phase transition between the high and the low density phases it has a pronounced jump from the left to the right density. A system size of 1000 is sufficiently large to localize the phase transition line. The transition into the maximal current phase is continuous, only the derivative of the order parameter has a (jump) discontinuity. In order to localize this phase transition line one needs larger systems of size 5000 .

In a given phase the simulated bulk density depends on the boundary densities the same way as it is predicted by the theory, namely it equals to the left (right) density in the LD (HD) phase, and it is a well defined constant value in the MC phase. At the phase boundary the crossover between two kinds of behavior of the bulk density can be localized within an error shown the figures (). The Monte Carlo results are seen to be in agreement with the phase diagram derived from the theory of boundary-

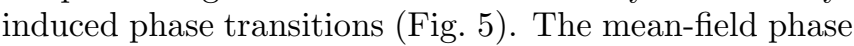
transition lines are significantly outside the numerical error bars except on part of the transition line between LD and MC phase.

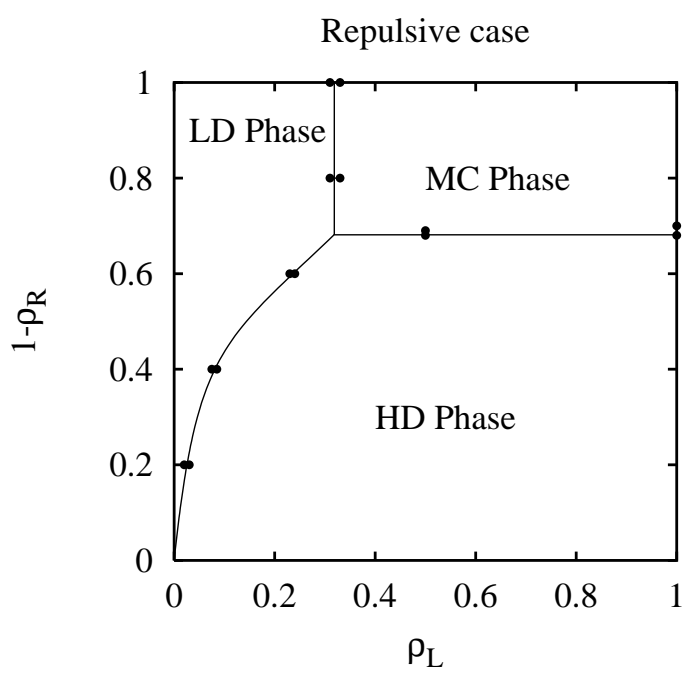

FIG. 5. Monte Carlo data and theoretical phase transition lines (full curves) for repulsive interaction. The numerical error bars are indicated by full circles.

\section{ATTRACTIVE INTERACTION}

Attractive (ferromagnetic) interaction leads to bulk particle domains as in the one-dimensional Ising model. Dynamically this is qualitatively understandable since because of $q>r$ particles tend to form clusters. One expects a shift of the maximal-current density $\rho^{*}$ to higher densities. This can indeed be shown by calculating the derivative of the exact current (7). The full currentdensity relation for $q=1, r=0.1$ is shown in Fig. 6 .

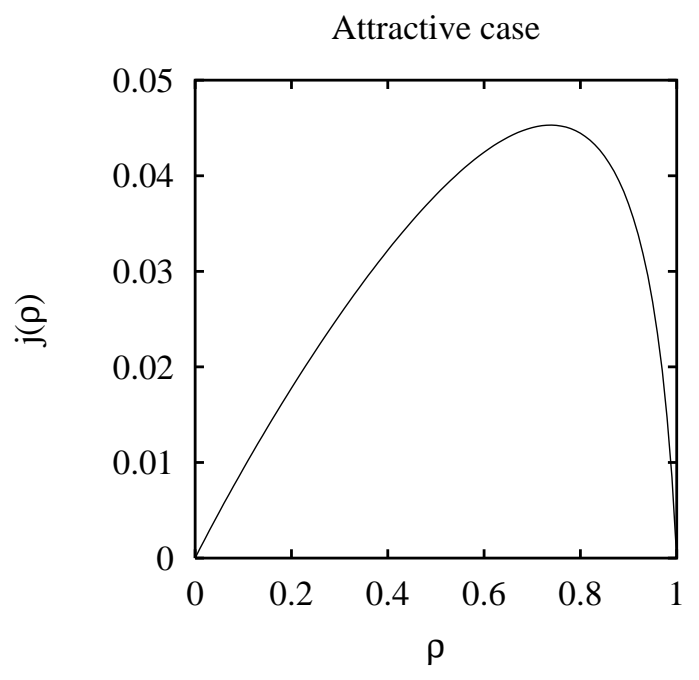


FIG. 6. Exact stationary current $j$ as a function of the density $\rho$ for $q=1, r=0.1$ (repulsive interaction).

Analysis of the model with open boundaries proceeds in the same way as in the repulsive case. With $r=0.1$ and $q=1.0$ the mean field approximation yields the three phases discussed above, with the phase transition lines differing substantially from those expected theoretically if one identifies $\rho_{-}=\rho_{L}$ and $\rho_{+}=\rho_{R}$ as suggested by the density profiles in the low and high density phases respectively.

As additional feature the mean field theory predicts a further phase transition located at the large $\rho_{L}$ and small $\rho_{R}$ corner of the phase diagram (Fig. 7). In this "fourth phase" the bulk density is larger than the density corresponding to the maximal current. The area of this phase increases with increasing attraction, and the area of MC phase decreases but does not disappear.

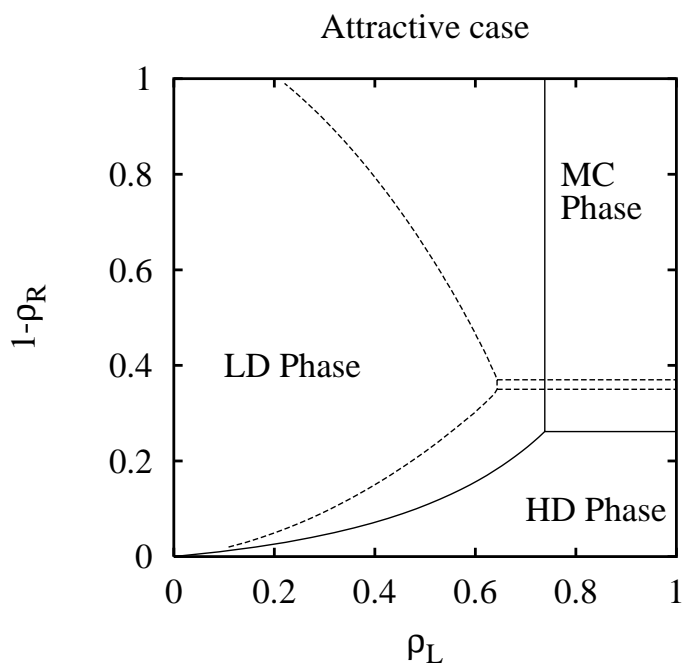

FIG. 7. Mean field phase diagram (broken curves) and theoretical phase transition lines (full curves) for repulsive interaction. The narrow area above the HD phase is the mean field MC phase. The fourth phase obtained from mean field covers the large area in the upper right part of the phase diagram.

The phase diagram obtained from Monte-Carlo simulation has the phase transitions expected from the theoretical prediction, but also shows that the fourth phase exists in a small neighborhood of the $\left(\rho_{+}=0, \rho_{-}>\rho^{*}\right)$ line and hence is not an artefact of the mean-field approximation. The location of the phase transition lines is rather different compared to mean field (Fig. 8). The transition to this phase from the LD phase is first order and from the maximal current phase it is second order. In this sense the fourth phase is like the high-density phase, however, the bulk density differs from both the boundary densities $\rho_{R, L}$. Within the theory of boundary-induced phase transitions this high density phase can be understood by recalling the non-universal relationship between real boundary density and effective boundary density (in terms of which the theory is formulated). While for repulsive interaction and the choice of coupling mechanism used here these two quantities may be identified, the connection is apparently more complicated for attractive interaction if the (real) boundary densities become sufficiently high (left boundary) and low (right boundary) respectively. An adequate explanation of this boundary phenomenon which leads to a reentrance transition into the high-density phase is not available. In order to rule out the possibility of a finite-size effect we performed simulations with different system sizes. Using $\mathrm{N}=500,1000$ and 5000 resp. we found no indication that the reentrance transition would disappear for large enough systems.

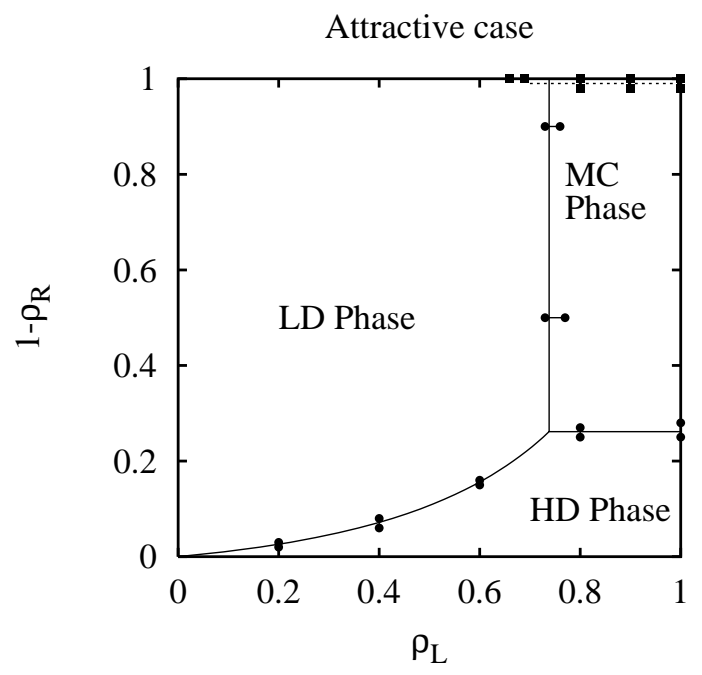

FIG. 8. Monte Carlo data and theoretical phase transition lines (full curves) for repulsive interaction. The numerical error bars are indicated by full circles. The reentrance phase transition to the fourth (HD) phase (upper right corner of the phase diagram) is marked by the broken curve interpolating between the Monte Carlo points.

\section{CONCLUSIONS}

Our analysis of the totally asymmetric exclusion model with next-nearest-neighbor interaction consists of two parts. First we considered the periodic system in order study the bulk properties of the steady state. With a view on traffic flow modelling we remark that the observations (i) - (iv) listed above are consistent with the results obtained here. The current-density relation becomes asymmetric (Fig. 1) in a way which is closer to real traffic data as the symmetric relation $j=\rho(1-\rho)$ for the ASEP with $r=q=1$. There is no discontinuity in the derivative at the maximal-current density $\rho^{*}$, in agreement with the arguments given above. As more far-reaching conclusion we note that the exact result (4) sheds light on the so far somewhat unclear relationship between the updating mechanism and the occurrence of 
correlations. In order to obtain correlations in traffic flow models it is not essential to use a discrete-time updating mechanisms. Since other discrete-time models have uncorrelated stationary states [28] it appears that correlations have their physical origin in speed-reduction rather than in the nature of the updating scheme.

In the second part we investigated the steady-state selection in the open system. It turns out that the theoretical scenario based on the interplay of shocks and overfeeding correctly describes the phase diagram in terms of effective boundary densities. For repulsive interactions this strengthens the case for a maximal current phase in real traffic. For attractive interaction there is, however, a surprising reentrance phase transition to the high density phase which originates in the so far poorly understood relationship between actual and effective boundary densities. Apparently these two are not always monotic functions of each other as one would naively expect. For a deeper understanding the next step to be done is the analysis of the density profiles close the reentrance phase transition lines in order analyze whether universal properties of the usual transition lines [3, 35, 10] can be observed or not.

T.A. thanks for partial support by the Hungarian Academy of Sciences (Grant OTKA T 029792). G.M.S. thanks K. Klauck, V. Popkov, L. Santen and A. Schadschneider for illuminating discussions on traffic flow.

\section{APPENDIX A: TRANSFER MATRIX FOR THE ONE-DIMENSIONAL ISING MODEL}

The energy of the one-dimensional Ising model with the classical spin variables $s_{i}= \pm 1$ can be written in term of the variables $n_{i}=\left(1-s_{i}\right) / 2$ in the form $E=J \sum_{i} n_{i} n_{i+1}$. In this interpretation the Ising model is a classical lattice gas of $M=\sum_{i} n_{i}$ hard-core particles with nearest neighbor interaction. Using standard techniques 32] one can express the grand canonical partition function $Z=\sum_{\text {config }} z^{M} e^{-\beta E}$ at inverse temperature $\beta=1 / k T$ in terms of the eigenvalues of the transfer matrix

$$
T=\left(\begin{array}{cc}
1 & \sqrt{z} \\
\sqrt{z} & z e^{-\beta}
\end{array}\right)
$$

where, for our model, $e^{-\beta J}=q / r$ and we define $z=$ $e^{-\beta h}$. In the spin interpretation of the model $h$ plays the role of a magnetic field.

The eigenvalues of the transfer matrix are

$$
\lambda_{1,2}=\frac{1}{2}\left(1+\frac{q}{r} z\right) \pm \sqrt{\frac{1}{4}\left(1+\frac{q}{r} z\right)^{2}+z\left(1-\frac{q}{r}\right)}
$$

where we choose $\lambda_{1}$ to the positive sign. For a system with $N$ sites and periodic boundaries one has $Z=$ $\operatorname{Tr} T^{N}=\lambda_{1}^{N}+\lambda_{2}^{N}$. The equilibrium distribution of the
Ising model is the stationary distribution of the particle hopping model. This does not mean that the particle hopping model reaches thermal equilibrium at long times, since the stationary distribution does not satisfy detailed balance with respect to the dynamics of the model. The non-equilibrium nature of the steady state results in a non-vanishing stationary particle current. We stress that completely different dynamical models may have the same stationary distribution, see e.g. the list of models given in 21. Another non-equilibrium example with the same Ising distribution is the discretetime ASEP with parallel update [31]. Even though the distribution is the same and hence all particle correlations are the same, the stationary current of this model 17,18 (which is defined via the dynamics by the continuity equation) is different from the current (6) in our model.

In order to calculate expectation values in the transfer matrix formalism we define the diagonal matrix

$$
n=\left(\begin{array}{ll}
0 & 0 \\
0 & 1
\end{array}\right) \text {. }
$$

The density is then given by $\rho=\operatorname{Tr}\left(n T^{N}\right) / Z_{N}$ and a two-point correlation function is given by $\left\langle n_{k} n_{l}\right\rangle=$ $\operatorname{Tr}\left(n T^{k-l} n T^{N-k+l}\right)$. Higher order correlators are calculated analogously. By diagonalizing $T$ one obtains the expression

$$
\rho=\frac{1-\lambda_{1}}{\lambda_{2}-\lambda_{1}}
$$

for the particle density in terms of the eigenvalues of $T$. This relation may be used to express the current (6) as a function of the particle density. In the thermodynamic limit $N \rightarrow \infty$ one obtains (7).

We note that expectation values for the distribution (16) are calculated with the same transfer matrix as in the periodic case. However, instead of taking a trace, one calculates a scalar product with suitably chosen vectors which are determined by the boundary fields.

\section{APPENDIX B: PROOF OF STATIONARITY FOR EQUAL BOUNDARY DENSITIES}

The proof of stationarity of the distribution (4) for the periodic system is given in Ref. [21]. An alternative, constructive proof can be obtained by using translational invariance and taking the distance between neighboring particles as stochastic variables. In this way the particle hopping process turns into a zero-range process [36] for which the stationary distribution is known. Reexpressing the stationary zero-range distribution in terms of particle occupation numbers yields (4).

Here we prove stationarity of the Ising distribution (16) for the open system coupled to reservoirs of equal density. We use a convenient standard approach for the stochastic description of classical interacting particle systems, 
known as "quantum Hamiltonian formalism" [5]. The basic idea is to formulate the generator of the Markov process in terms of a many-body quantum operator. For processes without exclusion one obtains in this way a Fock space representation of the generator in terms of bosonic creation and annilation operators 37 39. For exclusion processes with at most one particle per site the same strategy yields an operator expressed in terms of Pauli-spin matrices [5, 40 42].

We define the exclusion process with state space $X=$ $\{0,1\}^{N}$ and transition rates $w_{\underline{n} \rightarrow \underline{n}^{\prime}}$ from state $\underline{n}$ to $\underline{n}^{\prime}$ in terms of a master equation

$$
\frac{d}{d t} P(\underline{n} ; t)=\sum_{\underline{n}^{\prime} \in X}\left[w_{\underline{n}^{\prime} \rightarrow \underline{n}} P\left(\underline{n}^{\prime} ; t\right)-w_{\underline{n} \rightarrow \underline{n}^{\prime}} P(\underline{n} ; t)\right]
$$

for the probability $P(\underline{n} ; t)$ of finding, at time $t$, a configuration $\underline{n}$ of particles on a lattice of $N$ sites. Here $\underline{n}=\left\{n_{1}, n_{2}, \ldots, n_{N}\right\}$ where $n_{i}=0,1$ are the integervalued particle occupation numbers at site $i$. The master equation is a linear first order DGL in the time-variable and therefore it is natural to write it in a vector notation with the probabilities $P(\underline{n} ; t)$ as vector components. We represent each of the possible particle configurations $\underline{n}$ by a column vector $|\underline{n}\rangle$ which form a basis of a vector space $\mathbf{X}=\left(\mathbb{C}^{2}\right)^{\otimes N}$. The transposed vectors $\langle\underline{n}|$ form a basis of the dual space and we define the usual scalar product $\left\langle\underline{n} \mid \underline{n}^{\prime}\right\rangle=\delta_{\underline{n}, \underline{n}^{\prime}}$. The probability distribution is now represented by a state vector $|P(t)\rangle=\sum_{\underline{n} \in X} P(\underline{n} ; t)|\underline{n}\rangle$ and one can write the master equation in the form

$$
\frac{d}{d t} P(\underline{n} ; t)=-\langle\underline{n}|H| P(t)\rangle
$$

where the off-diagonal matrix elements of $H$ are the (negative) transition rates between states and the diagonal entries are the inverse of the exponentially distributed life times of the states. In formal analogy to the quantum mechanical Schrödinger equation we shall refer to $H$ as quantum Hamiltonian. A state at time $t^{\prime}=t_{0}+t$ is given in terms of an initial state at time $t_{0}$ by

$$
\left|P\left(t_{0}+t\right)\right\rangle=\mathrm{e}^{-H t}\left|P\left(t_{0}\right)\right\rangle .
$$

We stress that the physicists notion "quantum Hamiltonian" for the matrix $H$ is somewhat misleading in so far as $H$ is, in fact, the generator of the Markov semigroup of the process, rather than the Hamiltonian of an actual quantum system. This by now well-established notion has its origin in the fact that for various stochastic processes the generator $H$ is identical to the quantum Hamiltonian of some well-known spin system. In this context we would also like to point out that quantum mechanical expectation values $\langle A\rangle \equiv\langle\Psi|A| \Psi\rangle$ for an observable $A$ are calculated in a different way than probabilistic expectation values for a function $F(\underline{n})$ of the stochastic variables $\eta$. In the quantum Hamiltonian formalism one writes $\langle F\rangle \equiv \sum_{\underline{n} \in X} F(\underline{n}) P(\underline{n} ; t)=\langle s|F| P(t)\rangle$ with the matrix $F=\sum_{\underline{n} \in X} F(\underline{n})|\underline{n}\rangle\langle\underline{n}|$ and the summation vector $\langle s|=\sum_{n \in X}\langle\underline{n}|$ which performs the average over all possible final states of the stochastic time evolution.

For our considerations the expectation value $\rho_{k}(t)=$ $\left\langle s\left|n_{k}\right| P(t)\right\rangle$ for the density at site $k$ is of special interest. It is given by the projection operator $n_{k}$ which has value 1 if there is a particle at site $k$ and 0 otherwise. $m$-point density correlations are then given by the expression $\left\langle s\left|n_{k_{1}} \ldots n_{k_{m}}\right| P(t)\right\rangle$. In this paper we study only stationary expectation values. For the formal description of a stationary probability distribution we use the transposed summation vector $|s\rangle=\sum_{\underline{n} \in X}|\underline{n}\rangle$. A general stationary measure $P^{*}(\underline{n})$ may then be written in vector notation in the form $\left|P^{*}\right\rangle=e^{-\beta E(\underline{n})}|s\rangle / Z_{N}$ with the configuration-dependent "energy" matrix $E(\underline{n})$ and the "partition function" $Z_{N}=\left\langle s\left|e^{-\beta E(\underline{n})}\right| s\right\rangle$ which normalizes the measure $\left|\tilde{P}^{*}\right\rangle=e^{-\beta E(\underline{n})}|s\rangle$. Notice that in vector notation the expression $E(\underline{n})$ represents a diagonal matrix with the energies as diagonal elements. Below we shall also use the invertible diagonal matrix $P^{*}=e^{-\beta E(\underline{n})}$ which has the (unnormalized) stationary probabilities $P^{*}(\underline{n})$ as diagonal elements.

To obtain the quantum Hamiltonian for the time evolution of the interacting ASEP with open boundaries we note that one can represent any two-state particle system as a spin system by identifying a particle (vacancy) on site $k$ with a spin-down (up) state on this site. This allows for a representation of $H$ in terms of Pauli matrices where $n_{k}=\left(1-\sigma_{k}^{z}\right) / 2$ projects on states with a particle on site $k$ and $v_{k}=1-n_{k}$ is the projector on vacancies. The off-diagonal matrices $s_{k}^{ \pm}=\left(\sigma_{k}^{x} \pm i \sigma_{k}^{y}\right) / 2$ create $\left(s_{k}^{-}\right)$and annihilate $\left(s_{k}^{+}\right)$particles. We stress that in the present context the "spins" are just convenient labels for particle occupancies. Using this pseudospin formalism one finds

$$
\begin{aligned}
H= & \sum_{k=1}^{N-2}\left(n_{k} v_{k+1}-s_{k}^{+} s_{k+1}^{-}\right)\left(r n_{k+2}+q v_{k+2}\right) \\
& +\left(1-n_{1}-s_{1}^{-}\right)\left(\alpha_{1} n_{2}+\alpha_{2} v_{2}\right) \\
& +\beta_{1}\left(n_{N-1} v_{N}-s_{N-1}^{+} s_{N}^{-}\right)+\beta_{2}\left(n_{N}-s_{N}^{+}\right) .
\end{aligned}
$$

Within this formalism proof of stationarity is now a straightforward calculation, using simple expressions for the jumping rates at the ends:

$$
\begin{aligned}
\alpha_{1} & =\frac{q z}{\lambda_{1}\left(\lambda_{1}-1\right)} \\
\alpha_{2} & =\frac{r z}{\lambda_{1}\left(\lambda_{1}-1\right)} \\
\beta_{1} & =\frac{q z}{\lambda_{1}-1} \\
\beta_{2} & =\frac{q z}{\lambda_{1}} .
\end{aligned}
$$

and assuming that the boundary densities are equal at the two end. According to (B.3) stationarity is equivalent 
to the eigenvector relation $H\left|P^{*}\right\rangle=0$ which in turn is equivalent to

$$
\left(P^{*}\right)^{-1} H\left|\tilde{P}^{*}\right\rangle=\left(P^{*}\right)^{-1} H P^{*}|s\rangle=0 .
$$

The diagonal similarity transformation of $H$ with $P^{*}$ leads to a sum of transition matrices which act nontrivially on at most four neighboring sites. To calculate the action of the non-diagonal parts on $|s\rangle$ we use $s_{k}^{+}|s\rangle=v_{k}|s\rangle$ and $s_{k}^{-}|s\rangle=n_{k}|s\rangle$. This leaves only diagonal terms the sum of which vanishes identically. This proves stationarity of the measure (16).

[1] J. T. MacDonald, J. H. Gibbs, and A. C. Pipkin, Biopolymers 6, 1 (1968); J. T. MacDonald and J. H. Gibbs, Biopolymers 7, 707 (1969)

[2] T.M. Liggett, Trans. Amer. Math. Soc. 179, 433 (1975).

[3] J. Krug, Phys. Rev. Lett. 67, 1882 (1991).

[4] T.M. Liggett, Interacting Particle Systems, (Springer, Berlin, 1985); T.M. Liggett, Stochastic Interacting Systems, (Springer, Berlin, 1999).

[5] G. M. Schütz, Exactly solvable models for many-body systems far from equilibrium, to appear in: Phase Transitions and Critical Phenomena, C. Domb and J. Lebowitz (eds.) (Academic Press, London, 2000).

[6] see also the two contributions by S.A. Janowsky and J.L. Lebowitz (Chapter 13) and B. Derrida and M.R. Evans (Chapter 14) resp. in: Nonequilibrium Statistical Mechanics in One Dimension, V. Privman (ed.), (Cambridge University Press, Cambridge, 1997). For a connection to solitons in the continuum limit see H. Fogedby, Phys. Rev. Lett. 80, 1126 (1998).

[7] G. Schütz and E. Domany, J. Stat. Phys. 72, 277 (1993).

[8] B. Derrida, M. R. Evans, V. Hakim and V. Pasquier, J. Phys. A 26, 1493 (1993).

[9] G. Schütz, Phys. Rev. E 47, 4265 (1993).

[10] A.B. Kolomeisky, G.M. Schütz, E.B. Kolomeisky and J.P. Straley, J. Phys. A 31, 6911 (1998).

[11] V. Popkov and G.M. Schütz, Europhys. Lett. 48, 257 (1999).

[12] D. Chowdhury, L. Santen and A. Schadschneider, Curr. Sci. 77, 411 (1999). A more comprehensive review by the same authors will appear in Physics Reports (2000).

[13] D. Helbing, Verkehrsdynamik: Neue Physikalische Modellierungskonzepte, (Springer, Berlin, 1997).

[14] V. Popkov, L. Santen, A. Schadschneider and G.M. Schütz, preprint (1999).

[15] L. Neubert, L. Santen, A. Schadschneider and M. Schreckenberg, Phys. Rev. E 60, 6480 (1999).

[16] K. Nagel and M. Schreckenberg, J. Phys. I 2, 2221 (1992).

[17] A. Schadschneider and M. Schreckenberg, J. Phys. A 26, L679 (1993).

[18] M. Schreckenberg, A. Schadschneider, K. Nagel und N. Ito, Phys. Rev. E 51, 2939 (1995).

[19] V. Kukla, J. Kornatowski, D. Demuth, I. Girnus, H.
Pfeifer, L. Rees, S. Schunk, K. Unger, and J. Kärger, Science 272, 702 (1996).

[20] G. T. Barkema, C. Caron and J.F. Marko, Biopolymers 38665 (1996).

[21] S. Katz, J. L. Lebowitz and H. Spohn, J. Stat. Phys. 34, 497 (1984).

[22] F.L. Hall, L.A. Brian, M.A. Gunter, Transpn. Res. A 20, 3, 197 (1986).

[23] B.S. Kerner and H. Rehborn, Phys. Rev. E 53, R1297 (1996).

[24] K. Klauck and A. Schadschneider, Physica A 271, 102 (1999).

[25] J. Krug and H. Spohn, Phys. Rev. A 38, 4271 (1988).

[26] G. Schütz, J. Stat. Phys. 71, 471 (1993).

[27] S. Yukawa, M. Kikuchi, and S. Tadaki, J. Phys. Soc. Jap. 63, 3609 (1994).

[28] N. Rajewsky, L. Santen, A. Schadschneider and M. Schreckenberg, J. Stat. Phys. 92, 151 (1998).

[29] L.G. Tilstra and M.H. Ernst, J. Phys. A 31, 5033 (1998).

[30] J. de Gier and B. Nienhuis, Phys. Rev. E 59, 4899 (1999).

[31] H. Yaguchi, Hiroshima Math. J. 16, 449 (1986).

[32] R. J. Baxter, Exactly solved models in statistical mechanics, (New York, Academic Press, 1982).

[33] T. Nagatani, J. Phys. A 28, 7079 (1995).

[34] H. Spohn, Large Scale Dynamics of Interacting Particles, (Springer, Berlin, 1991).

[35] K. Oerding and H. K. Janssen, Phys. Rev. E 58, 1446 (1998).

[36] F. Spitzer, Adv. Math. 5, 246 (1970).

[37] L. P. Kadanoff and J. Swift, Phys. Rev. 165, 310 (1968).

[38] M. Doi, J. Phys. A 9, 1465 (1976).

[39] P. Grassberger and M. Scheunert, Fortschr. Phys. 28, 547 (1980).

[40] E. Siggia, Phys. Rev. B 16, 2319 (1977)

[41] S. Sandow and S. Trimper, Europhys. Lett. 21, 799 (1993).

[42] F. C. Alcaraz, M. Droz, M. Henkel and V. Rittenberg, Ann. Phys. (NY) 230, 250 (1994). 